\title{
Post-traumatic osteomyelitis: an unresolved conundrum in orthopedic trauma surgery
}

\author{
P. F. Stahel ${ }^{1}$ C. Mauffrey ${ }^{1}$
}

Received: 28 June 2016 / Accepted: 7 July 2016 / Published online: 21 July 2016

(C) Springer-Verlag Berlin Heidelberg 2016

The management of post-traumatic infections remains arguably one of the last frontiers in the field of orthopedic trauma surgery. Despite growing interest by specialty societies and research funding bodies, clinical innovation on the topic is hampered by inadequate risk stratification, large variability in clinical presentation, and the widespread use of "off-label" treatment strategies that lack FDA approved indications. Current diagnostic tools fall short of adequate sensitivity to allow early detection and timely management of post-traumatic osteomyelitis. For example, the use of polymerase chain reaction (PCR)-based technology allows earlier detection of microbial DNA in sonication fluid with higher sensitivity than conventional bacterial cultures, yet PCR is costly and not widely available for routine diagnostics. The need to implant foreign materials for early fracture stabilization represents an unresolved conundrum due to the vulnerability of orthopedic implants to bacterial colonization and subsequent infections. Despite advances in sterile precautions, staged damage-control protocols with scheduled "2nd look" procedures and the availability of new generation antibiotics and sophisticated microsurgical techniques for soft tissue coverage, successful strategies to prevent and mitigate post-injury infection rates remain elusive. This special issue of the European Journal of Trauma and Emergency Surgery (EJTES) is dedicated to innovations in diagnostic work-up and management strategies for post-traumatic osteomyelitis.

P. F. Stahel
philip.stahel@dhha.org

1 Department of Orthopedics, Denver Health Medical Center and University of Colorado School of Medicine, 777 Bannock Street, Denver, CO 80204, USA
In the first paper, Govaert and Glaudemans provide an encompassing overview on the work-up of suspected post-traumatic osteomyelitis by nuclear medicine testing modalities, including single-photon emission computed tomography (SPECT) and positron emission tomography (PET) [1]. The intuitive advantage of these testing modalities over standard imaging lies in the notion that nuclear medicine reflects the physiological function of the system being investigated (e.g., local glucose metabolism or leukocyte accumulation as surrogate markers of infection), as opposed to the exclusive anatomical imaging by traditional tests, such as CT or MRI. The article by Govaert and Glaudemans [1] furthermore addresses the value of new and innovative hybrid imaging options (SPECT-CT, PETCT, PET-MRI) with higher sensitivity for early detection of either acute or chronic low-grade infections. These new diagnostic modalities have been largely neglected in the field of orthopedic trauma and represent an intriguing opportunity for future research in an important and evolving field.

The second article by Mauffrey et al. provides a segue from diagnostics to improved pathophysiogical understanding of the enigmatic "biofilm" on orthopedic implants and its underlying therapeutic ramifications for eradication of post-injury osteomyelitis [2]. New treatment options in clinical practice are, furthermore, discussed in the third article by Tosounidis and colleagues, which elaborate on the role of the reamer-irrigator-aspirator (RIA) technique in the management of post-operative long bone infections [3]. In their excellent article, the authors discuss advantages as well as limitations and potential complications related to the use of RIA as an adjunct to post-operative infection management [3].

The cutting-edge topics related to diagnostics, pathophysiology, and management of post-traumatic orthopedic 
infections presented in this special EJTES issue reflect an exciting and insightful snapshot of the current state of the art and emerging advances in the field. Persisting challenges, limitations, and barriers to improving patient safety and patient outcomes will hopefully ignite the interest of the next generation of enthusiastic academic researchers and clinicians in pursuing preventive and curative measures to minimize and extinguish the daunting threat of post-traumatic infections imposed on our patients today.

\section{Compliance with ethical standards}

The authors comply with the ethical guidelines for authorship and publishing in the European Journal of Trauma and Emergency Surgery. This article does not contain any studies with human or animal subjects performed by the authors.
Conflict of interest Philip F. Stahel and Cyril Mauffrey declare no conflicts of interest in relation to this work.

\section{References}

1. Govaert GAM, Glaudemans AWJM. Nuclear medicine imaging of posttraumatic osteomyelitis. Eur J Trauma Emerg Surg. 2016. doi:10.1007/s00068-016-0647-8.

2. Mauffrey C, Herbert B, Young H, et al. The role of biofilm on orthopaedic implants: the "Holy Grail" of post-traumatic infection management? Eur J Trauma Emerg Surg. 2016. doi:10.1007/ s00068-016-0694-1.

3. Tosounidis TH, Calori GM, Giannoudis PV. The use of Reamerirrigator-aspirator in the management of long bone osteomyelitis: an update. Eur J Trauma Emerg Surg. 2016. doi:10.1007/ s00068-016-0700-7. 